\title{
Uterine Artery Embolization of Symptomatic Uterine Fibroids: Success in Short Term Results
}

\author{
Dr. Saeeda Bin Salam Bahakam ${ }^{1}$, Dr. Anila Aravindan ${ }^{2 *}$, Dr. Anupama Bondili ${ }^{3}$, Dr. Jamal Aldeen AlKoteesh ${ }^{4}$ \\ ${ }^{1,}$ Resident in Obstetrics \& Gynaecology - Tawam Hospital, Al Ain, United Arab Emirates \\ ${ }^{3}$ Consultant Obstetrics and Gynaecology, Consultant Urogynecology- Tawam Hospital, Al Ain, United Arab Emirates \\ ${ }^{4}$ Consultant Radiology - Tawam Hospital, Al Ain, United Arab Emirates
}

\begin{abstract}
DOI: $10.36348 /$ sijog.2021.v04i04.012 $\quad$ | Received: 24.02.2021 | Accepted: 10.04.2021 | Published: 22.04 .2021
*Corresponding author: Dr. Anila Aravindan

Abstract
\end{abstract}

To evaluate the efficacy of uterine artery embolization (UAE) for the treatment of symptomatic uterine fibroids in patients treated at $\mathrm{Al}$ Ain Hospital, United Arab Emirates. Medical records and radiological images of all patients undergoing UAE in Al-Ain Hospital between 1 January 2012 and 31 December 2017 were retrospectively analysed. 141 patients aged 23-50 years were included in the study. Single femoral access technique was used in all patients. Clinical improvement was assessed by questioning patients regarding symptomatic improvement and follow up MRI to see the reduction in size of the dominant leiomyoma. Data was analyzed using SPSS 22. All 141 patients underwent technically successful UAE. 55 were lost follow up and were not included into the final analysis. Out of the remaining 85 patients, $55.3 \%$ were nulliparous and $44.7 \%$ were multiparous. A significant number of females that is $70.5 \%$ had multiple uterine fibroids compared to $30 \%$ who had only single uterine fibroid. $71.7 \%$ patients had menorrhagia pre-operatively, $98.4 \%$ had complete resolution at 6 months. As per the size reduction, paired t-Test showed statistically significant reduction in the mean leiomyoma size from $8 \mathrm{~cm}$ to $6 \mathrm{~cm}$ and then further to $5 \mathrm{~cm}$ at 6 months and 1 year respectively. Almost $91.8 \%$ of the patients who underwent UAE were satisfied with the improvement in their symptoms as an outcome compared to 8.2\% who were unsatisfied. Uterine fibroid embolization represents a promising method of treating fibroid-related menorrhagia and pelvic pain. Further studies with larger number of patients and long-term follow-up are needed.

Keywords: Fibroids, Uterine artery embolization, Menorrhagia, Pelvic pain, Leiomyoma, Dysmenorrhea.

Copyright () 2021 The Author(s): This is an open-access article distributed under the terms of the Creative Commons Attribution 4.0 International License (CC BY-NC 4.0) which permits unrestricted use, distribution, and reproduction in any medium for non-commercial use provided the original author and source are credited.

\section{INTRODUCTION}

Uterine Fibroids are known to be the most prevalent benign tumors of the uterus among females of childbearing age. They are composed of smooth muscle and fibrous connective tissue whose growth is dependent on estrogen and progesterone [1,2].

Their occurrence is age dependent and the incidence is high in the reproductive age group with significant reduction in post-menopausal women. They can be identified in about $80 \%$ of women by the age of 50 [3]. Among those 20 to $50 \%$ are symptomatic fibroids whereas the remaining are asymptomatic, usually found incidentally during a routine ultrasound [4].

Demographically, prevalence of fibroids is higher in African women when compared to White and Asian women [5].
Some of the prominent clinical features of fibroids include dysmenorrhea, abnormal uterine bleeding, abdominal distension, pressure symptoms including urinary frequency, constipation and dyspareunia [6]. These set of symptoms can cause significant effect on the quality of life of patients [7]. Most women troubled with these symptoms of fibroid, seek treatment [6].

According to the guidelines of fibroid treatment, management should only be offered to women with a symptomatic or rapidly growing fibroid. Current available options for treatment include surgical and non-surgical approach $[8,9]$.

With regards to surgical management, myomectomy is the preferred in women who wished to preserve their uterus, however an increased incidence of recurrence and re-intervention has been reported with 
this procedure. In women with severe symptoms, hysterectomy has been offered as definitive treatment; however it too is associated with increased risk of infection and morbidity [8, 9].

Patient opting for medical management have the option of gonadotrophin releasing hormone $(\mathrm{GnRH})$ analogues which is effective in reducing size and symptoms associated with fibroids but its side effects like hot flushes, vaginitis, bone loss and breast reduction prevents its long term use [9]. Now newer modalities for non-surgical interventions have evolved, of them Uterine Artery Embolization (UAE) has gained recognition as an effective treatment choice [10]. In comparison to long term gonadotrophin releasing hormone $(\mathrm{GnRH})$ analogues UAE has fewer side effects [11].

Uterine artery embolization (UAE) has been approved by the National Institute for Clinical Excellence (NICE) as a non-invasive treatment option for uterine fibroids due to its efficiency in relieving symptoms [12]. The main mechanism of action is inhibiting the growth of fibroids by limiting its blood supply. Since the first UAE was introduced in 1995, a large number of international researches has been conducted to understand its efficacy and safety in clinic practice $[13,14]$. It is hence, established as an optimal choice for patients who desire relief from the symptoms of fibroid but who wish to preserve their uterus or avoid surgery due to medical contraindication or personal preferences[10].

Among the recognized advantages of Uterine Artery Embolization the most substantial one is that it is a non-surgical uterine sparing treatment option with significant reduction in fibroid size and symptomatic improvement $[14,5]$. It is also associated with reduced hospital stay and enables early return to routine life and therefore, it also has a positive impact on the psychology and Quality of Life (QoL) of patients [8].

Unfortunately, there have also been few drawbacks for UAE including post-operative embolization syndrome that is abdominal cramps due to degeneration of fibroid, infection at site of catheter insertion and few patient experience iatrogenic amenorrhea post procedure [8].

A few absolute contraindications for UAE are fibroids that are asymptomatic, pregnancy, suspicion or diagnosed uterine malignancy, active pelvic inflammatory disease, allergy to the radiological contrast agents and renal insufficiency $[12,8]$.

Several relative contraindications have been mentioned in various studies among them the most controversial one is future fertility, few studies are against UAE for patient desiring future pregnancy whereas, the American College of Obstetricians and
Gynecologists states that the effect of UAE on pregnancy remains understudied but makes no recommendation of whether desire for a future pregnancy is a contraindication [15].

Apart from that, it is not considered the treatment of choice in postmenopausal women. Fibroids in post-menopausal women reduce significantly in size and symptoms hence presence of large sized uterus in post-menopausal women raises the suspicion of malignancy and need for further investigation and follow up. So this treatment is essentially reserved for premenopausal women with symptomatic fibroids [8].

Characteristics of the fibroid also play a part while offering UAE, for example for a pedunculated fibroid of subserosal or submucosal origin with a narrow stalk (stalk $<50$ percent in diameter in relation with the largest diameter fibroid), may detach and lead to sterile peritonitis or may cause intra uterine infections $[15,12]$. According to literature if the size of fibroid is large it is advised that such cases should not undergo UAE, as it is associated with increased pain, infection and post embolization syndrome. However, the exact threshold of size or number of fibroids has not been established. So far there was one study that brought the series of cases with a dominant fibroid of $>10 \mathrm{~cm}$ and/or a uterine volume of $>700 \mathrm{~cm}$ and no increased risk of serious complications was concluded $[15,16]$.

In this study we aimed to evaluate that UAE is a safe and reliable alternative to surgery for symptomatic fibroid in our institution.

\section{METHODOLOGY}

Study: This is a retrospective cohort study conducted from 1 January 2012 to 31 December 2017 in Alain hospital. Institutional ethical approval has been taken for the study from the local ethics committee at Alain hospital

Subject: This study included 141 patients who underwent Uterine Artery Embolization for symptomatic uterine fibroids between the ages of 23 to 54 with imaging showing fibroids: single/multiple/subserosal, submucosal, intramural, and pedunculated.

Patients were advised to have clinical and Imaging (MRI) follow up at 6 months and 1 year. Compliance of patients regarding follow up, however, was suboptimal and 55 patients were lost to follow up. Medical records and radiological images of all patients were retrospectively analyzed. Patients $(n=55)$ who were lost to follow up were excluded from the final analysis. Results were statistically analyzed using Statistical Package for the Social Sciences (SPSS) 22.0. Descriptive statistical Method was used using Chi square test. In addition, paired t-Test was used for 
analyzing fibroid size reduction. All patients attended outpatient follow-up at six months and one-year postprocedure, at which time follow-up radiography (Magnetic Resonance Imaging) was performed. The information was entered in a forum designed especially for the study.

The site of study: Al Ain Hospital is fully accredited hospital by Joint Commission International, serves as a teaching and referral medical institute supporting and having close collaboration with the College of Medicine and Health Sciences at United Arab Emirates University. With more than 300 doctors and 35 specialties at $\mathrm{Al}$ Ain Hospital, that treats nearly 20,000 in-patients and 320,000 outpatients every year, as well as 350 emergency cases per day. The Obstetrics and Gynaecology Department contains 48 beds and performs around 3,000 deliveries per year. Regarding interventional radiology, Alain hospital is the largest center in the United Arab Emirates, patient from all over the country come for treatment here around 5000 procedures are done over 1 year ( including elective /emergency /obstetric and general). The department has 2 consultants and 1 interventional radiology suite and outpatient clinic twice a week.

\section{Procedure}

In all patients, right femoral arterial access was used for embolizing both uterine arteries. A 5-Fr pigtail catheter placed at L1 level was used for flush aortogram initially to detect any aberrant supply to the uterus. Another flush run was carried out with the tip of the pigtail catheter at the level of bifurcation of aorta. A 5Fr uterine artery catheter (Roberts Uterine or Cobra; Cook Incorporated, Bloomington, IN, USA) was advanced into each uterine artery. Embolization was carried out through 2.9 microcatheter (Contata; Cook Incorporated, Bloomington, IN, USA) with poly vinyl alcohol (PVA) particles of 355-500 mm, with the tip of catheter lying in the horizontal portion of the artery. Stasis of contrast column was taken as end-point of embolization; care was also taken to avoid reflux and nontarget embolization. The postembolization angiograms were carried out in all cases, after withdrawing the tip of the catheter in the vertical or proximal horizontal portion of the uterine artery, to confirm adequate embolization. In 3 patients, in addition to uterine arteries, a left ovarian artery seen to supply the fibroid was also embolized. For analgesia during the procedure we perform Superior hypogastric nerve block by identifying the Aortic bifurcation first then inserting $22 \mathrm{G}$. Chiba needle which is advanced to contact anterior surface of L5 vertebral body anteriorly and confirmed under Fluoroscopy guidance. Once the tip is in the proper position, a small amount of contrast with lidocaine is injected to verify the needles are in the correct spot outside the vessels and safely situated adjacent to the superior hypogastric plexus .If the target spot verify, then followed by $20 \mathrm{ml}$ Ropivacaine $0.75 \%$ mixed with $5 \mathrm{ml}$ of contrast connected through long connecting tubing.

After the procedure, diclofenac sodium injection (Tromax; Aristo Pharmaceuticals, Mumbai) and pethidine injection (Verpat, Verve Healthcare, Ankleshwar, India) intramuscularly were used. These patients were discharged on the same day evening with oral antibiotics and analgesics.

\section{RESULTS}

Among the 85 patients, $43.5 \% \quad(n=37)$ were single and $56.5 \%(\mathrm{n}=48)$ were married. Around 47 $(55.3 \%)$ were nulliparous and $38(44.7 \%)$ were Multiparous. Majority of Patients $57 \% \quad(n=48)$ Complained of Dysmenorrhea pre-operatively. Almost $72 \%$ of women with fibroids had menorrhagia. Around 31 patients had significant abdominal distension caused by the fibroids compared to 54 who didn't. $22 \%(n=19)$ of females had increased urinary frequency due to fibroids. A significant number of females $(n=60)$ had multiple Uterine Fibroids compared to 26 who had only single uterine fibroid. $70 \%$ of Patients had mild postprocedural pain compared to $25 \%$ having moderate pain, while only $3.5 \%$ had severe pain.

Table-1: Study Population and Presenting Signs \& Symptoms.

\begin{tabular}{|l|l|}
\hline Patient characteristic & Number (\%) \\
\hline Age & $48(56.5 \%)$ \\
\hline Younger than 40 & $37(43.5 \%)$ \\
\hline Older or more than 40 & \\
\hline Marital Status & $37(43.5 \%)$ \\
\hline Single & $48(56.5 \%)$ \\
\hline Married & \\
\hline Parity & $47(55.3 \%)$ \\
\hline Nulliparous & $38(44.7 \%)$ \\
\hline Multiparous & $48(56.5 \%)$ \\
\hline Pre-operative dysmenorrhea & $37(43.5 \%)$ \\
\hline Present & \\
\hline Absent & $61(71.8 \%)$ \\
\hline Pre-operative menorrhagia & $24(28.2 \%)$ \\
\hline Present & $31(36.5 \%)$ \\
\hline Absent & $54(63.5 \%)$ \\
\hline Pre-operative Abdominal Distension \\
\hline Present & $19(22.4 \%)$ \\
\hline Absent & $66(77.6 \%)$ \\
\hline Pre- operative Urinary frequency \\
\hline Present & $25(29.4 \%)$ \\
\hline Absent & $60(70.6 \%)$ \\
\hline Number of Fibroids & \\
\hline Single & \\
\hline Multiple & \\
\hline Total Patients n=85 & \\
\hline
\end{tabular}


Post-Operative Follow-up

Table-2: Post-Operative Signs and Symptoms at 6 Months and 1 Year Follow-up

\begin{tabular}{|l|l|l|}
\hline Symptoms & 6 Months & 1 Year \\
\hline Post - Dysmenorrhea & $\mathrm{n}=48$ & $\mathrm{n}=24$ \\
\hline Resolved & $24(50 \%)$ & $17(70.8 \%)$ \\
\hline Improved & $24(50 \%)$ & $7(29.2 \%)$ \\
\hline Post - Menorrhagia & $\mathrm{n}=61$ & $\mathrm{n}=61$ \\
\hline Resolved & $60(98.4 \%)$ & $56(91.8 \%)$ \\
\hline Reduced but heavier & $1(1.6 \%)$ & $5(8.2 \%)$ \\
\hline $\begin{array}{l}\text { Post Abdominal } \\
\text { Distension }\end{array}$ & $\mathrm{n}=31$ & $\mathrm{n}=31$ \\
\hline Reduced & $24(77.4 \%)$ & $16(51.6 \%)$ \\
\hline No change & $5(16.1 \%)$ & $15(48.4 \%)$ \\
\hline Unknown & $2(6.5 \%)$ & - \\
\hline $\begin{array}{l}\text { Post Urinary } \\
\text { Frequency }\end{array}$ & $\mathrm{n}=19$ & $\mathrm{n}=8$ \\
\hline Improved & $8(42.1 \%)$ & $1(12.5 \%)$ \\
\hline Resolved & $11(57.9 \%)$ & $7(87.5 \%)$ \\
\hline Total Patients $\mathbf{n}=85$ & & \\
\hline
\end{tabular}

Out of 61 patients who had menorrhagia preoperatively, $60(98.4 \%)$ had complete resolution at 6 months while the remaining patient had reduced but heavier bleeding. At 1-year follow-up, there was a relapse of 5 patients $(8.2 \%)$ who had reduced but heavier menstruation.

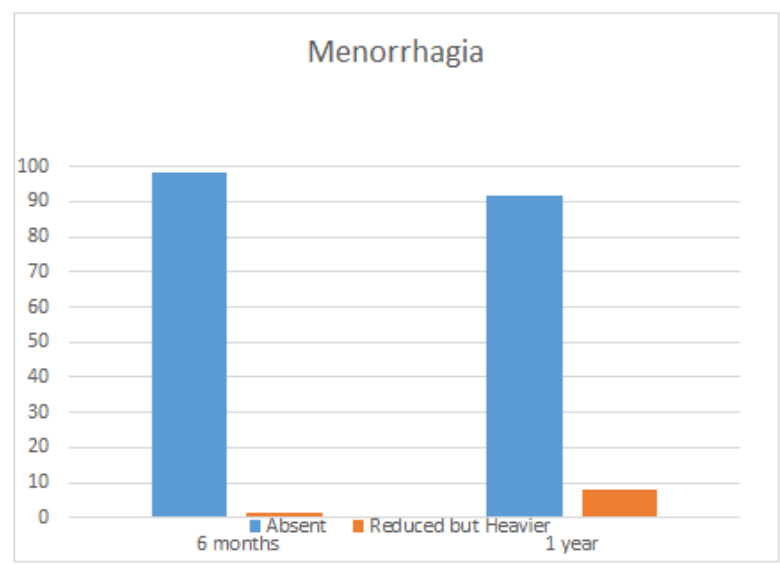

Graph-1: Post-Operative Menorrhagia

Among Patients who have complained of dysmenorrhea $n=48$, almost $50 \%(n=24)$ had improved symptoms, moreover, another $50 \%$ had complete resolution of dysmenorrhea at 6 months. Among those who still had residual dysmenorrhea at 6 months, $70 \%$ $(n=17)$ had complete resolution of symptoms at 1 year follow up.

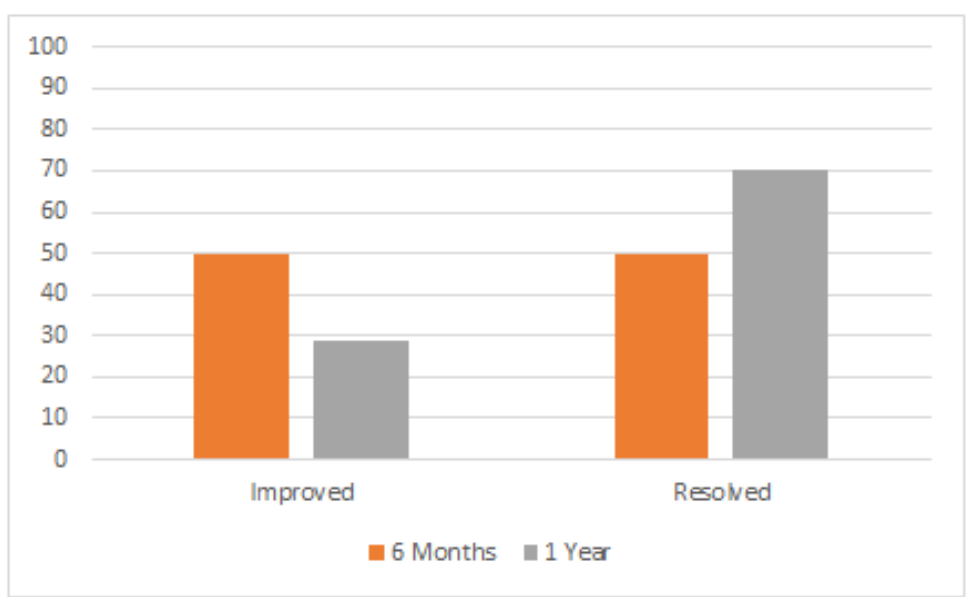

Graph-2: Post-operative Dysmenorrhea

From those patients who presented with Abdominal Distension, $77 \%$ had reduction in abdominal circumference at 6 months with $51 \%$ of them having further reduction at 1 year and $48 \%(n=15)$ having complete resolution of distension.

Among the 19 patients who initially complained of urinary frequency, $58 \%(\mathrm{n}=11)$ had complete resolution and $42 \% \quad(\mathrm{n}=8)$ had improved symptoms. After 1 year of UAE, 7 more had complete resolution of urinary frequency.
One of the main outcomes of this study is the reduction in size of the fibroid post UAE. In this study MRI was used as the modality of choice to estimate the size of fibroid pre-procedure, at 6 months and at 1 year follow up. The Largest diameter was used as a landmark to calculate the size reduction by using the following equation:

$$
\% \text { Reduction }=\frac{\text { Initial diameter preop }- \text { largest diameter postop }}{\text { Initial diameter }} \times 100
$$

The percentages were further classified into below $30 \%, 31-50 \%, 50.1-80 \%$, and more than $80 \%$ reduction. The data were as follows: 
Table-3

\begin{tabular}{|l|l|l|}
\hline \% Reduction & At 6 months & At 1 year \\
\hline$<30 \%$ & $56(65.9 \%)$ & $35(41.2 \%)$ \\
\hline $30-50 \%$ & $25(29.4 \%)$ & $26(30.6 \%)$ \\
\hline $50.1-80 \%$ & $4(4.7 \%)$ & $23(27.1 \%)$ \\
\hline$>80 \%$ & 0 & $1(1.2 \%)$ \\
\hline
\end{tabular}

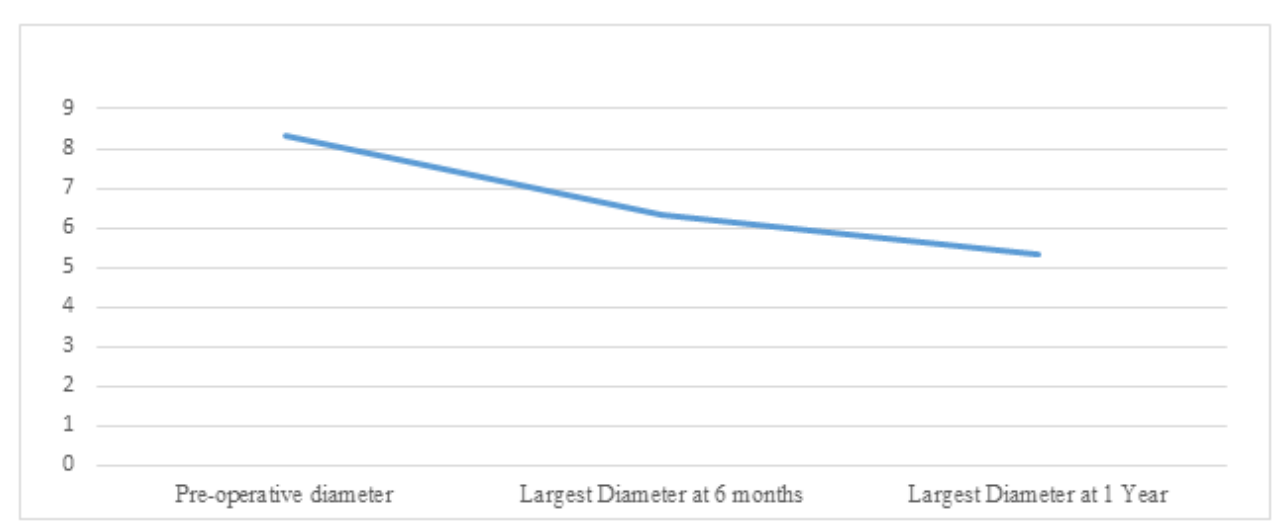

Graph-3: Mean Diameter Reduction in Fibroid size

As per the size reduction, paired t-Test was done to compare the mean of largest diameter preoperatively to the mean of the largest diameter at 6 months and 1 year respectively. The Results showed statistical significance $(\mathrm{P}=.000)$ in which there was a reduction of the mean leiomyoma size from $8 \mathrm{~cm}$ to $6 \mathrm{~cm}$ and then further to $5 \mathrm{~cm}$.

Almost $91.8 \% \quad(n=78)$ of the patients who underwent UAE were satisfied by the outcomes compared to $8.2 \%(n=7)$ were unsatisfied.

The need for secondary intervention was spared in $79(92 \%)$ of the patients while 6 patients required secondary intervention including repeat UAE $\mathrm{n}=3$, Myomectomy $\mathrm{n}=2$, and 1 patient required hysterectomy 3 years after the procedure.

The majority $72.9 \% \quad(n=62)$ of UAE procedures were conducted as a day case while $20 \%$ $(n=17)$ stayed for 1day post-op, 5 remained for 2 days and 1 patient remained for 3 days due to severe postoperative pain.

\section{DISCUSSIONS}

Minimally invasive techniques have become one of the leading approaches in treating Uterine Fibroids. The main aim in managing symptomatic uterine fibroids is the reduction of dominant fibroid size therefore decreasing overwhelming symptoms of menorrhagia, dysmenorrhea, urinary frequency, and abdominal distension. This will lead to improving the quality of life [14].

As UAE has gained popularity through the last decade and because our facility is one of the leading institutions for UAE in the country, patients sought medical care to undergo the procedure in order to avoid major surgery. Therefore, our sample size of 85 was superior to other studies with almost same study duration. For example, in a study conducted in Pakistan [9] the initial sample size was only 14 patients included during a 6 years study period.

Patients with symptomatic fibroids who underwent UAE within our facility who wished to preserve their uterus and avoid major surgery. They were mainly referred to our facility by the gynecologist along with few who opted for the procedure due to its growing popularity.

One of the primary outcomes of our study was the effect of UAE on menorrhagia. According to Jawad et al. [9] there was $77 \%$ reduction in menorrhagia symptoms and $89 \%$ reduced dysmenorrhea. On the other hand, in a study conducted in Denmark [17] $96 \%$ of patients had decreased bleeding, $70 \%$ experienced lesser pain, and $61 \%$ had reduced bulk symptoms at 6 months follow-up. Compared to our study, results were almost equivalently successful in which we had $98.4 \%$ complete resolution of menorrhagia at 6 months in patients with menorrhagia pre-operatively.

In Anderson et al. [17] Al, the main method of follow-up for uterine fibroid size was ultrasonography whereas in our study Magnetic Resonance Imaging was used, which is the most effective diagnostic modality for visualizing the size and location of uterine fibroids. In addition, it is best reserved for procedural planning for complicated procedures such as UAE.

The second major primary outcome in this study is the size reduction of uterine fibroids. In Odense University Hospital [17] it was found that the mean volume reduction of fibroid size was $68 \%$ at 6 months follow-up. While in Georgetown University [18] 
Saeeda Bin Salam Bahakam et al; Sch Int J Obstet Gynec, Apr. 2021; 4(4): 149-155

Hospital the mean percent volume reduction of leiomyomas was $43.6 \%$ at 3 months and $57.8 \%$ at 12 months. Furthermore, according to a similar study conducted in Galway, Ireland [19] there was a mean reduction of $27 \%$ and $68 \%$ of the dominant leiomyoma size at 3 and 12 months consistently. Comparatively we had a mean reduction of $25 \%$ and $37 \%$ at 6 months and 1 year respectively.

Although, scientific literature doesn't recommend embolization of very large fibroids due to the increased risk of complications and May further predispose to secondary hysterectomy, there was no limit to the leiomyoma size in our study. The largest fibroid was $23 \mathrm{~cm}$ in diameter and there was around $26 \%$ reduction in size at 1 year. Similarly, in Anderson et al, [17] it was reported successful embolization of large fibroids up to $726 \mathrm{~cm} 3$ in size.

Compared to Rischbieter et al. [14] who had $2.4 \%(2 / 82)$ repeat embolization 12 -18 months after the primary intervention, we had $3.5 \%(3 / 85)$ rate of repeat embolization. However, the overall success rate was rewarding.

One of the limitations of our study was the overall duration of follow-up in addition to the loss of follow-up rates. In our study patients were followed up at 6 months and 1 year, whereas in Spies et al. [18] the patients were followed up to 5 years post embolization, which reflected more insight of the long-term effect of the procedure. In addition, we had only $60 \%$ follow-up rate compared $91 \%$ in the latter study.

In Iglesias et al. [7] around 44\% of patient's experienced mild pain post-op while $46 \%$ experienced severe pain. However, in our study $70 \%$ of patients had mild post- procedural pain compared only $3.5 \%$ had severe pain. In addition, overall patient satisfaction was high.

Our 6 months to 12 months follow up confirms the efficacy along with safety in management of symptomatic fibroids using UAE. Due to the expertise of the operator, technical failure was none.

\section{CONCLUSION}

Uterine artery embolization is a safer noninvasive uterine sparing approach with positive impact in the quality of life of women with symptomatic uterine myomas who are not eligible or do not desire to undergo surgical management.

It is a method with a high technical success rate. The treatment has good response on fibroid volume reduction and clinical symptom associated with lower morbidity, shorter hospital stay and recovery time. Complications and adverse events are otherwise few or rare.
Further research is clearly necessary to see long term response and future need of intervention.

\section{REFERENCES}

1. Bulun, S.E. (2013). Uterine fibroids. N Engl J Med, 369(14):1344-1355.

2. Ishikawa, H., Ishi, K., Serna, V. A., Kakazu, R., Bulun, S. E., \& Kurita, T. (2010). Progesterone is essential for maintenance and growth of uterine leiomyoma. Endocrinology, 151(6), 2433-2442.

3. Zimmermann, A., Bernuit, D., Gerlinger, C., Schaefers, M., \& Geppert, K. (2012). Prevalence, symptoms and management of uterine fibroids: an international internet-based survey of 21,746 women. BMC women's health, 12(1), 1-11.

4. Vilos, G. A., Allaire, C., Laberge, P. Y., Leyland, N., Vilos, A. G., Murji, A., \& Chen, I. (2015). The management of uterine leiomyomas. Journal of Obstetrics and Gynaecology Canada, 37(2), $157-$ 178.

5. Gupta, J. K., Sinha, A., Lumsden, M. A., \& Hickey, M. (2014). Uterine artery embolization for symptomatic uterine fibroids. Cochrane Database of Systematic Reviews, (12).

6. Bukulmez, O., \& Doody, K. J. (2006). Clinical features of myomas. Obstetrics and Gynecology Clinics, 33(1), 69-84.

7. García-Iglesias, Á., Perez, S. G., Vaquero, D. B., Martin, M. D. L. O. R., Valle, S. G., Escanilla, J. L. P., .. \& Picaso, J. B. (2013). Uterine artery embolization for the treatment of uterine fibroids. Electronic Journal of Biomedicine, (1).

8. De La Cruz, M. S. D., \& Buchanan, E. M. (2017). Uterine fibroids: diagnosis and treatment. American family physician, 95(2), 100107.

9. Jawad, R. A., Rana, A. I., \& Tariq, N. (2013). Uterine fibroid embolisation: Initial experience in our local population. JPMA. The Journal of the Pakistan Medical Association, 63(12), 1482-1485.

10. Volkers, N. A., Hehenkamp, W. J., Birnie, E., de Vries, C., Holt, C., Ankum, W. M., \& Reekers, J. A. (2006). Uterine artery embolization in the treatment of symptomatic uterine fibroid tumors (EMMY trial): periprocedural results and complications. Journal of vascular and interventional radiology, 17(3), 471-480.

11. Aubuchon, M., Pinto, A. B., \& Williams, D. B. (2002). Treatment of uterine fibroids. Primary care update for OB/GYNS, 9(6), 231-237.

12. National Institute for Health and Care Excellence (NICE). (2010). Uterine artery embolization for fibroids. availiable at: http://

www.nice.org.uk/IPG367

13. Ravina, J. H., Ciraru-Vigneron, N., Bouret, J. M., Herbreteau, D., Houdart, E., Aymard, A., \& Merland, J. J. (1995). Arterial embolisation to treat uterine myomata. The Lancet, 346(8976), 671-672.

14. Rischbieter, P., Sinclair, C., Lawson, A., \& Ahmad, S. (2016). Uterine artery embolisation as 
an effective choice for symptomatic fibroids: Fiveyear outcome. SA Journal of Radiology, 20(1).

15. Clinical Recommendations on the use of uterine artery embolization in the management of fibroids, Retrieved $\quad 14 \quad$ April $2020 \quad$ from https://www.rcog.org.uk/globalassets/documents/g uidelines/23-12-2013_rcog_rcr_uae.pdf

16. Smeets, A. J., Nijenhuis, R. J., van Rooij, W. J., Weimar, E. A., Boekkooi, P. F., Lampmann, L. E., ... \& Lohle, P. N. (2010). Uterine artery embolization in patients with a large fibroid burden: long-term clinical and MR followup. Cardiovascular and interventional radiology, 33(5), 943-948.
17. Andersen, P. E., Lund, N., Justesen, P., Munk, T., Elle, B., \& Floridon, C. (2001). Uterine artery embolization of symptomatic uterine fibroids: initial success and short-term results. Acta Radiologica, 42(2), 234-238.

18. Spies, J. B., Bruno, J., Czeyda-Pommersheim, F., Magee, S. T., Ascher, S. A., \& Jha, R. C. (2005). Long-term outcome of uterine artery embolization of leiomyomata. Obstetrics \& Gynecology, 106(5 Part 1), 933-939.

19. Laios, A., Baharuddin, N., Iliou, K., Gubara, E., \& O'Sullivan, G. (2014). Uterine artery embolization for treatment of symptomatic fibroids; a single institution experience. Hippokratia, 18(3), 258. 